\title{
Environnement précoce et vulnérabilité neuropsychiatrique
}

$>$ Du fait de son immaturité à la naissance, le nouveau-né est totalement dépendant des soins parentaux pour sa survie et pour son développement cérébral. Des altérations de la qualité de l'environnement précoce, non seulement lors de l'enfance, mais également en période prénatale, ont un impact, à long terme, sur la santé. Récemment, chez l'animal, des travaux ont mis en évidence des modulations épigénétiques après des stress précoces qui pourraient se transmettre sur plusieurs générations, via des modifications dans les cellules germinales. <

Il existe des périodes critiques durant lesquelles l'organisme est fortement influencé par son environnement (Figure 1). L'adversité précoce est associée à de nombreux problèmes de santé à l'âge adulte et augmenterait le risque de développer des troubles neuropsychiatriques tels que la dépression ou l'état de stress post-traumatique, mais également une obésité ou des maladies cardiovasculaires [1]. L'adversité précoce est généralement définie comme l'exposition à des maltraitances dans l'enfance, telles que des négligences parentales, des abus sexuels, physiques ou psychologiques. Avant et après la naissance, d'autres facteurs comme la maladie, la malnutrition ou l'exposition à des drogues constituent aussi des stress précoces importants. À ce titre, l'environnement socio-économique pendant le développement, par la pluralité des aspects qu'il intègre, est un facteur déterminant pour l'état de santé psychique et physique de l'enfant devenu adulte [2]. Dans cet article nous présenterons les recherches montrant les conséquences de stress précoces (pendant l'enfance et in utero) sur la santé mentale chez l'homme et sur les comportements, dans les modèles animaux. Nous illustrerons également des mécanismes épigénétiques associés au stress précoce.

\author{
Marion Rincel ${ }^{1,2}$, Amandine Lépinay ${ }^{1,2}$, \\ Anne Gabory ${ }^{3}$, Vassilia Théodorou ${ }^{4}$, Muriel Koehl ${ }^{5}$, \\ Valérie Daugé ${ }^{6}$, Stefania Maccari ${ }^{7,8}$, \\ Muriel Darnaudéry ${ }^{1,2}$
}

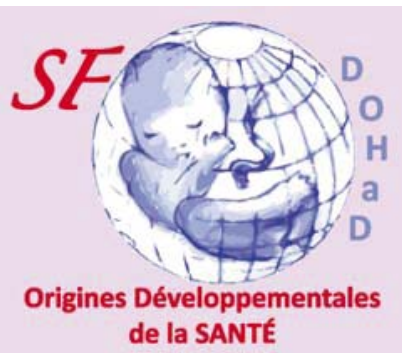

\section{Adversité durant \\ l'enfance et vulnérabilité neuropsychiatrique: données cliniques et modèles animaux de stress psycho-social}

Chez beaucoup de mammifères, les petits naissent immatures et totalement dépendants des soins parentaux. L'immaturité du cerveau les rend très sensibles à leur environnement. Depuis les recherches menées par James Harlow, René Spitz et John Bowlby, père de la théorie de l'attachement, il est admis que le développement cognitif et affectif de l'enfant requiert la mise en place d'un lien affectif privilégié avec une figure d'attachement (le parent ou son substitut). Des enfants exposés à des stress sévères tels que des carences affectives précoces ou des

${ }^{1}$ Université de Bordeaux, Nutrition et neurobiologie intégrée (NUTRINEURO), UMR 1286, 146, rue Léo Saignat, 33076 Bordeaux Cedex, France ;

${ }^{2}$ Inra, Nutrition et neurobiologie intégrée (NUTRINEURO), UMR 1286, F-33076 Bordeaux, France;

${ }^{3}$ UMR 1198, biologie du développement et reproduction, Inra - Centre de recherche de Jouy en Josas, F-78352 Jouy-en-Josas, France ; ${ }^{4}$ Inra, UMR 1331- Toxicologie alimentaire (TOXALIM), F-31027 Toulouse, France ; ${ }^{5}$ Neurocentre Magendie, université Bordeaux, F- 33077 Bordeaux, France;

${ }^{6}$ Inra, Microbiologie de

l'alimentation au service de la Santé (MICALIS), UMR 1319, équipe Alimentation, microbiote intestinal, pathologies encéphaliques et métabolique (AMIPEM) F-78352, Jouy-en-Josas, France ; ${ }^{7}$ Sapienza university of Rome/

Istituto di Ricovero e Cura a Carattere Scientifico (IRCCS), Istituto Neurologico Merditerraneo (NEUROMED), Italie ;

${ }^{8}$ UMR 8576 CNRS, université de Lille, France. muriel.darnaudery@ u-bordeaux.fr maltraitances parentales présentent des atteintes neurodéveloppementales de structures clefs dans la régulation des processus cognitifs et émotionnels (atrophie du cortex préfrontal et de l'hippocampe, atrophie ou hypertrophie de l'amygdale, selon les études). À l'âge adulte, ces effets peuvent perdurer et s'accompagner d'altérations de 


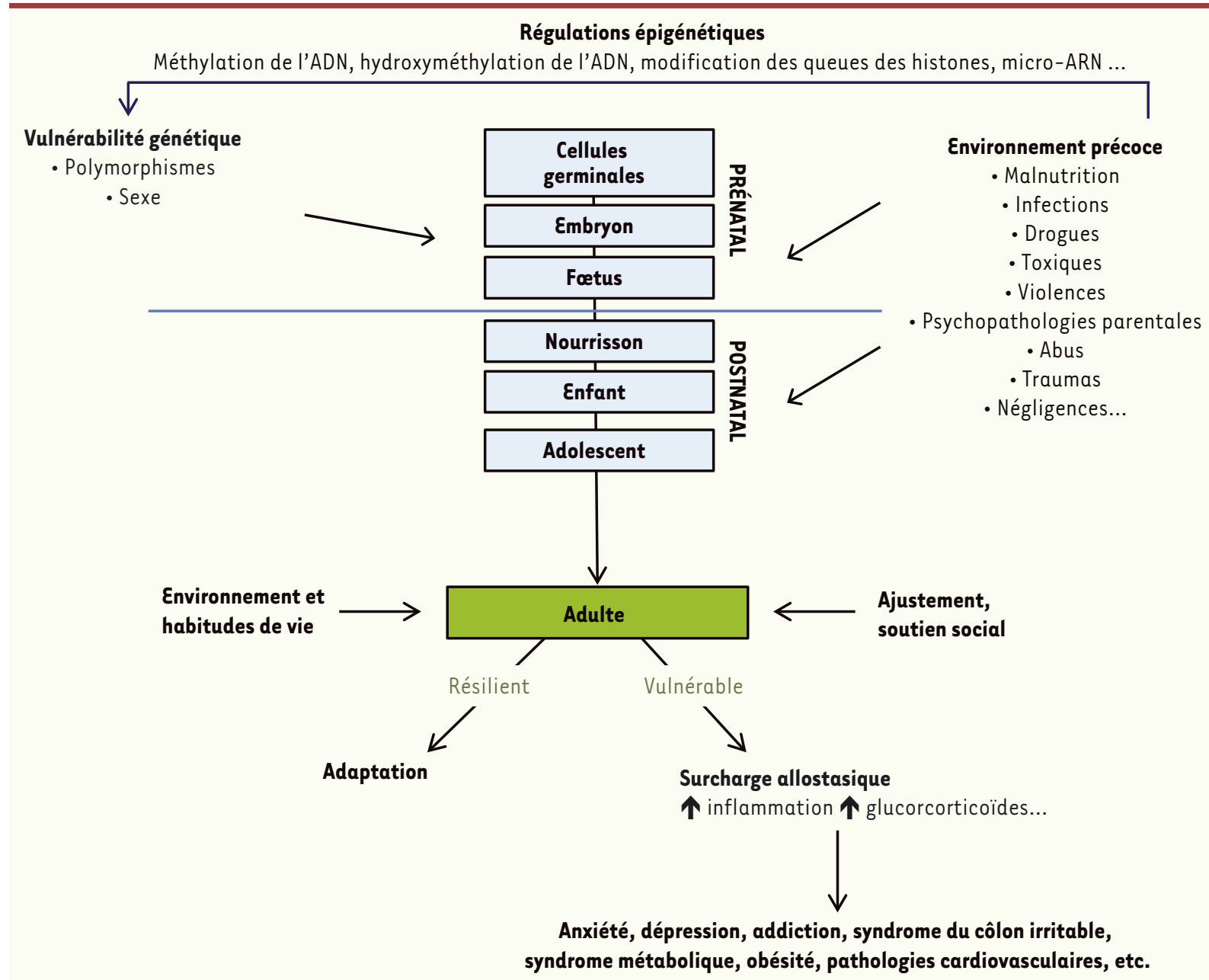

Figure 1. Stress précoces, surcharge allostasique et vulnérabilité à l'âge adulte. Les premières phases de vie constituent une période critique lors de laquelle les facteurs environnementaux laissent une empreinte durable, suggérant ainsi un phénomène de « programmation précoce » du phénotype adulte. Ainsi, l'adversité précoce, se présentant sous de multiples formes telles que la malnutrition ou encore des violences parentales, augmente la vulnérabilité aux troubles neuropsychiatriques et métaboliques à l'âge adulte. Les régulations épigénétiques représentent un mécanisme majeur pour expliquer l'effet de l'environnement sur l'individu en développement. De plus, la vulnérabilité de l'individu adulte dépend également de son contexte génétique propre. Un contexte génétique inadapté et une pression environnementale trop forte aboutiront à un état de rupture de l'homéostasie physiologique ou surcharge allostasique, lequel est caractérisé par une incapacité d'adaptation à de nouveaux stress.

l'axe corticotrope (système neuroendocrinien du stress) et d'un risque plus élevé de troubles neuropsychiatriques (Figure 2) [3, 4].

Chez le rongeur, les travaux princeps menés par Seymour Levine ont mis en évidence des périodes critiques durant lesquelles l'environnement inscrit une empreinte durable sur le cerveau [5]. La femelle rat « materne » de façon intense ses petits (allaitement actif en position arc-boutée, léchage ano-génital) ; ce soin maternel est déterminant pour la maturation cérébrale et les futures capacités adaptatives de la progéniture. II a été montré qu'à l'âge adulte les descendants des mères à faible maternage présentent des altérations comportementales, une réponse exacerbée de l'axe corticotrope face à un stress et une diminution des récepteurs des glucocorticoïdes dans l'hippocampe (Figure 3) [2]. En outre, l'adoption de ratons issus de mères biologiques à fort maternage, par des mères à faible mater- nage, produit un phénotype semblable à celui observé chez la descendance de mères à faible maternage. Enfin, après des adoptions croisées, la descendance femelle présente, à l'âge adulte, le même comportement maternel que celui de la mère adoptive [6]. Chez le rat, la séparation répétée mères-petits pendant de longues périodes produit, chez la descendance, un phénotype proche de celui induit par le faible maternage : altérations de l'axe corticotrope, perturbations émotionnelles (hyperanxiété, anhédonie) et mnésiques (Figure 3) [7]. Ces changements sont associés, entre autres, à des altérations morphofonctionnelles dans le cortex préfrontal médian et l'hippocampe (diminution de l'arborisation et du nombre d'épines dendritiques 


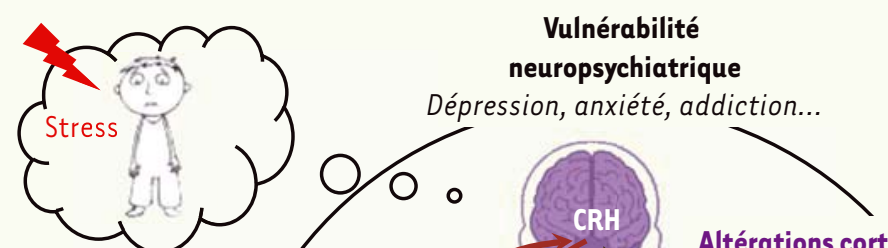

Changements de la méthylation de I'ADN

- GR (cortex, hippocampe, sang, placenta)

- BDNF (cortex préfrontal)

- AVP (hippocampe, hypothalamus)

- NR4Al (hippocampe)

- Era (hypothalamus)

- FKBP5 (sang)

- MeCP2 (striatum et cellules germinales mâles)

- CBI (cellules germinales mâles)

- CRH-R2 (cellules germinales mâles)

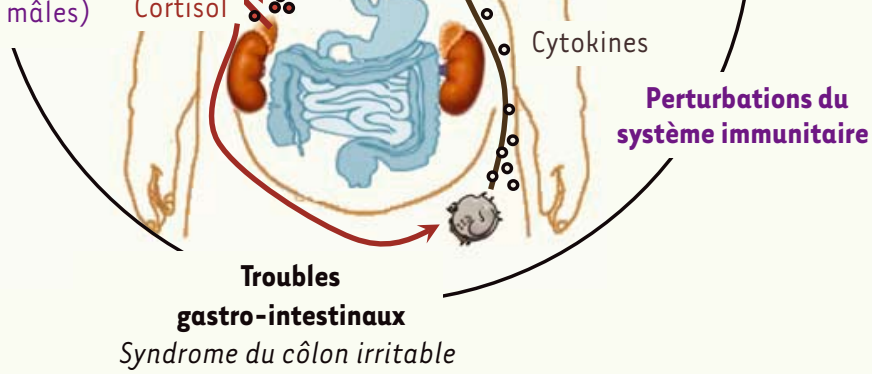

Figure 2. Changements physiologiques à l'âge adulte associés à l'adversité dans l'enfance et mécanismes épigénétiques potentiellement impliqués. L'adversité précoce est associée à des altérations de divers systèmes physiologiques tels que le système nerveux central et périphérique, le système immunitaire ou encore le système gastrointestinal. Ces perturbations physiologiques sont accompagnées de modifications épigénétiques comme la méthylation ou la déméthylation de l'ADN dans les régions promotrices de nombreux gènes (les changements observés chez l'homme sont en rouge gras, les autres changements ont été observés chez l'animal). L'ensemble de ces altérations pourrait participer à l'émergence de pathologies neuropsychiatriques. CRH : corticotropin releasing hormone; ACTH : adrenocorticotropic hormone; GR : glucocorticoid receptors; BDNF : brain derived neurotrophic factor ; AVP : arginin vasopressin ; NR4Al : nuclear receptor subfamily 4, group A, member 1 ; Era : estrogen receptor alpha ; FKBP5 : FK506 binding protein 5 ; MeCP2 : methyl CPG binding protein 2 ; CB1 : cannabinoid receptor type 1; CRH-R2 : corticotropin releasing hormone receptor 2.

des neurones pyramidaux, perturbations de la potentialisation à long terme ${ }^{1}$ ). La carence maternelle produit, dans I'hippocampe adulte, une diminution du facteur neurotrophique BDNF (brain derived neurotrophic factor) et des altérations des récepteurs du glutamate, NMDA ( $N$-methyl-D-aspartate receptor). Elle induit par ailleurs une hypersensibilité aux effets récompensant des drogues d'abus et une hypoactivité du système enképhalinergique ${ }^{2}$ dans les noyaux caudéputamen et accumbens [8]. Les systèmes opioïdes sont très impliqués dans la mise en place des relations entre la mère et le petit, et leur dysfonctionnement pourrait être responsable des altérations comportementales des rats stressés. D'autres perturbations de l'environnement postnatal précoce, comme la réduction de la disponibilité du matériel de nidification, ont également des effets délétères à long terme sur la descendance. En revanche, des séparations mèrespetits de courtes durées (15 min par jour) ont des effets opposés,

${ }^{1}$ La potentialisation à long terme est caractérisée par une amélioration durable de l'efficacité synaptique. Ce phénomène est généralement associé à la notion de plasticité neuronale et de mémoire.

${ }^{2}$ Les enképhalines sont des endorphines (morphine endogène) qui se fixent sur des récepteurs opioïdes. Elles jouent un rôle en particulier dans l'inhibition des messages de douleur et la régulation des réponses de stress. caractérisés par un phénotype résilient attribué à la stimulation du comportement maternel [9].

Par ailleurs, de nombreuses études ont montré que l'adversité précoce constitue un facteur de risque à l'émergence de troubles gastro-intestinaux, en particulier du syndrome du côlon irritable ${ }^{3}$ (Figure 2). En effet, il existe une communication bidirectionnelle cerveau-intestin, fortement influencée par le stress. La période néonatale est caractérisée par une grande plasticité neuronale des circuits sensitifs somatiques viscéraux. Chez le rongeur, la séparation maternelle affecte de façon durable l'homéostasie intestinale. Les conséquences majeures sont: (1) des modifications de l'immunité de la muqueuse intestinale, (2) l'apparition d'une hypersensibilité viscérale, et (3) l'altération de la fonction de la barrière de l'intestin. Ces animaux

${ }^{3}$ Appelé aussi syndrome de l'intestin irritable, il est caractérisé par une douleur
abdominale chronique et des désordres de la fonction intestinale (diarrhée ou / et constipation). 


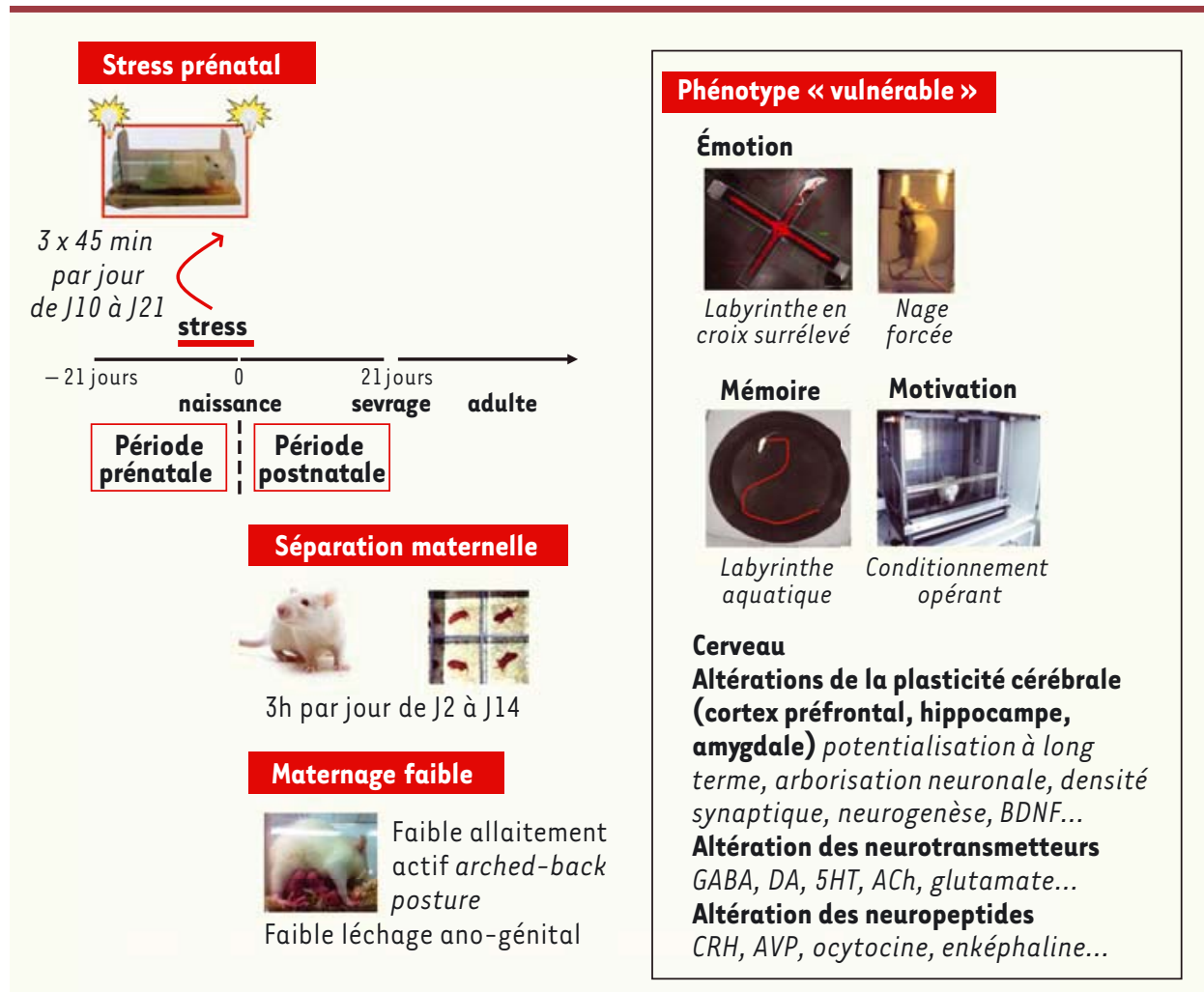

Figure 3. Exemples de modèles de stress précoces chez l'animal produisant un phénotype vulnérable à l'âge adulte. Dans des tests précliniques, les animaux exposés aux stress précoces présentent des altérations émotionnelles (caractérisées, en autres, par une faible exploration des bras ouverts dans le labyrinthe en croix surélevé et une forte immobilité dans le test de la nage forcée). Ces effets sont atténués par un traitement avec des antidépresseurs. Les animaux exposés aux stress précoces présentent également des altérations cognitives, en particulier une diminution des capacités d'apprentissage et de mémoire dans des tâches spatiales dépendantes de l'hippocampe. Enfin les stress précoces exacerbent la motivation pour les drogues d'abus. Sur le plan neurobiologique, le stress précoce affecte la plasticité cérébrale, les systèmes de neurotransmetteurs et de nombreux neuropeptides. BDNF : brain derived neurotrophic factor; GABA : gamma-aminobutyric acid; DA : dopamine ; 5HT : 5-hydroxytryptamine (sérotonine); ACh : acétylcholine ; $\mathrm{CRH}$ : corticotropin releasing hormone; AVP : arginin-vasopressin.

présentent à l'âge adulte une augmentation du nombre des mastocytes et des polynucléaires neutrophiles muqueux, ainsi qu'une réponse locale cytokinique lymphocytaire de type Th1 ( $T$ helper) et Th2 témoignant d'un tonus inflammatoire intestinal à bas bruit ${ }^{4}$ [10]. Plus récemment il a été montré que la susceptibilité à une hypersensibilité viscérale des animaux soumis à la séparation maternelle peut être transmise à la génération suivante.

\section{Qu'en est-il du stress avant la naissance?}

Pendant la grossesse, l'environnement utérin constitue à la fois une protection contre les agressions externes, et, via le placenta, un système de « conduction » qui relie le fœtus à son futur environnement. L'état physique de la mère détermine fortement le développement du fœtus qu'elle porte $(\rightarrow)$.

L'exposition à certains médicaments, drogues d'abus, virus, toxines microbiennes peut affecter l'embryon via leur passage placentaire et avoir des effets irrémédiables. De plus, les procédures d'assistance médicale à la procréation, comme la $(\rightarrow)$ Voir les Synthèses de B. Le Magueresse et al., et de P. Chavatte-Palmer et al., pages 51 et 57 de ce numéro fécondation in vitro suivie du transfert d'embryons, ou les traitements hormonaux aboutissant à une superovulation, ont des conséquences

${ }^{4}$ Activation chronique des processus immunitaires mais à un niveau relativement faible. potentielles à long terme sur la descendance, qui restent encore mal connues. Enfin, la question du rôle de l'état émotionnel de la mère sur le développement du fœtus a aussi été posée. Cependant, la démonstration d'un lien causal demeure difficile à établir, du fait de la lourdeur des études prospectives et des biais associés aux études rétrospectives. Depuis de nombreuses années, Vivette Glover étudie l'incidence du stress maternel lors de la grossesse sur le devenir de l'enfant [11]. Certains travaux rapportent une augmentation du risque de troubles émotionnels et cognitifs chez l'enfant, et des retards de langage. Ces effets sont en partie indépendants de causes pouvant survenir après la naissance de l'enfant, comme une dépression de la mère.

Chez l'animal, dès les années 1960, des études ont suggéré que le comportement de la descendance pouvait être modulé par des changements hormonaux de l'environnement intra-utérin ou par les expériences vécues par la femelle lors de la gestation, voire même avant la conception [12]. Depuis, une littérature abondante a porté sur le stress gestationnel chez le rongeur et son incidence sur la descendance [13, 14]. Chez le rat, un stress prénatal (contention maternelle lors de la 
gestation, Figure 3) provoque, chez la progéniture, des changements biochimiques et comportementaux durables, qui reflètent l'induction d'une programmation pathologique précoce. À l'âge adulte, lors d'un nouveau stress, les mâles ayant subi un stress prénatal présentent une sécrétion prolongée de corticostérone, caractéristique d'un déficit de rétrocontrôle négatif de l'axe corticotrope [15]. Sur le plan comportemental, l'exposition de la mère à un stress pendant la gestation produit chez la progéniture (1) une augmentation des comportements de types anxieux et dépressifs, (2) une augmentation de la motivation et/ou de la sensibilité aux drogues d'abus [13] et (3) des atteintes cognitives (retard d'apprentissage spatial, déficit de consolidation de la mémoire de peur, déficits de mémoire de travail). Une partie des déficits comportementaux associés au stress prénatal pourrait impliquer l'hippocampe. En effet, sur le plan neurobiologique, le stress prénatal entraîne des atteintes de la plasticité hippocampique, telles que la suppression de la potentialisation à long terme, l'atrophie des neurones granulaires et pyramidaux ou encore une réduction de la neurogenèse adulte [16], un effet certainement lié à l'hyperactivité chronique de l'axe corticotrope. Ces altérations sont accompagnées d'une baisse de l'expression des récepteurs métabotropiques [17] et ionotropiques (NMDA [acide $\mathrm{N}$-méthyl-D-aspartique] et AMPA [ $\alpha$-amino-3-hydroxy-5-méthylisoazol-4-propionate]) du glutamate dans I'hippocampe [18]. De façon intéressante, un bon nombre des altérations comportementales et neurobiologiques que l'on observe chez l'animal adulte sont atténuées par les antidépresseurs [19, 20]. Étant donnés les liens existant entre axe corticotrope et stress [15], la programmation à long terme des effets du stress prénatal sur la descendance pourrait impliquer les glucocorticoïdes. Le stress de contention provoque une activation de l'axe corticotrope et une augmentation des taux circulants de corticostérone chez la femelle gestante (Figure 3 ). En condition normale, le fœtus est protégé par la $11 \beta$ HSD2 (11 $\beta$-hydroxystéroïde déhydrogénase de type 2 ), une enzyme qui inactive la corticostérone. Cependant, le stress maternel altère l'expression placentaire et l'activité de cette enzyme [21]. De plus, la surrénalectomie, réalisée chez les mères stressées, atténue certains effets sur la descendance [22].

Le stress gestationnel est vraisemblablement associé à un stress postnatal. En effet, l'exposition à des stress pendant la gestation perturbe le comportement de maternage, ce qui, comme on l'a vu précédemment, détermine les capacités d'adaptation au stress de la descendance. Par ailleurs, des manipulations postnatales précoces (adoption, stimulations tactiles, etc.) réduisent les altérations provoquées par le stress prénatal [23].

\section{Stress précoces et épigénome}

L'étude princeps concernant la programmation épigénétique et le stress précoce est parue en 2004. Elle montrait que les effets du faible maternage sur la descendance mettaient en jeu des régulations épigénétiques de la région promotrice du gène codant le récepteur des glucocorticoïdes ( $\mathrm{Nr} 3 \mathrm{cl}$ [nuclear receptor subfamily 3 , group C, member 1]) dans l'hippocampe (Figure 2). En effet, le faible maternage produit dans cette structure une augmentation de la méthylation de I'ADN et une diminution de l'acétylation des histones, lesquelles induisent une diminution de la transcription pouvant aboutir à des altérations comportementales et endocriniennes [24]. Ces altérations sont atténuées par des infusions intracérébroventriculaires d'un inhibiteur d'histone déacétylases (HDAC) à l'âge adulte. Les régulations épigénétiques associées à l'adversité précoce concernent aussi d'autres gènes tels que ceux codant le BDNF (brain derived neurotrophic factor), la vasopressine, le CRH (corticotropin releasing hormone) ou le récepteur $\mathrm{CRH}-\mathrm{R} 2$ (corticotropin releasing hormone receptor 2) [4] (Figure 2). La plus grande sensibilité aux opiacés des rats exposés à une séparation maternelle implique aussi des processus épigénétiques. En effet, la séparation maternelle produit une augmentation de la MeCP2 (methyl-CpG-binding protein) et de l'histone déacétylase HDAC2, ainsi qu'une diminution de l'acétylation des histones $\mathrm{H} 3$ et $\mathrm{H} 4$ dans les noyaux caudé-putamen et accumbens. De manière intéressante, le comportement de dépendance et l'hypoactivité du système enképhalinergique sont supprimés par le valproate de sodium, un inhibiteur d'histone déacétylases [25]. Certaines des altérations épigénétiques décrites chez l'animal sont présentes chez l'homme (Figure 2). Ainsi, une augmentation de la méthylation de la région promotrice du gène $\mathrm{Nr} 3 \mathrm{cl}$ a été observée dans l'hippocampe de suicidés (souffrant de dépression) ayant subi des maltraitances dans l'enfance [3]. Par ailleurs, chez l'animal comme chez l'homme, des études révèlent des changements de méthylation de I'ADN dans de nombreuses régions du génome après des stress postnataux précoces, que ce soit dans le système nerveux central (hippocampe, cortex préfrontal) ou dans les tissus périphériques (cellules sanguines, salive, épithélium buccal) [4, 25]. Le stress in utero est également associé à des changements épigénétiques: (1) une baisse de l'expression placentaire de la $11 \beta$ HSD2 (qui inactive la corticostérone), accompagnée d'une augmentation de la méthylation de I'ADN dans les sites $3 C p G$ du promoteur du gène codant cette enzyme et (2) des changements de méthylation de l'ADN dans les régions promotrices des gènes codant le $\mathrm{CRH}$ et le récepteur des glucocorticoïdes [27]. Cependant, des régulations épigénétiques opposées sont rapportées en fonction de l'intensité du stress (stress léger: augmentation de la méthylation de I'ADN ; stress fort : diminution de la méthylation) et du sexe (HDAC4 augmentée dans I'hippocampe des mâles; diminuée dans le cortex préfrontal des femelles). 
Les effets délétères de l'adversité précoce semblent transmis à travers les générations [28] $(\rightarrow)$. $(\rightarrow)$ Voir la Synthèse de C. Junien et al., page 35 de ce numéro
Les processus potentiellement impliqués dans la transmission du stress parental à la descendance sont multiples, mais récemment il a été proposé que le stress puisse agir sur les cellules germinales. Chez la souris, plusieurs études ont rapporté une transmission intergénérationnelle par le père. Ainsi, un stress chronique chez des souris mâles produit des changements neurocomportementaux qui se maintiennent chez sa descendance ( $F 1$ ) et à la $2^{e}$ génération (F2). Ces changements pourraient être transmis via des marques épigénétiques dans le sperme. En effet, une diminution de la méthylation de l'ADN dans la région promotrice du gène $C R H-R 2$ et une hyperméthylation des sites $C p G$ du promoteur du gène MeCP2 ont été observées dans le sperme des Fl et F2.

\section{Conclusion-perspectives : promouvoir le bien-être précoce pour favoriser la résilience?}

Depuis plus de 50 ans, les recherches sur l'adversité précoce soulignent ses conséquences sur la santé mentale et la santé en général. Des travaux récents rapportent des changements épigénétiques que l'on observe à l'âge adulte après une exposition à des stress lors de la période de développement. II faut cependant souligner qu'une part non négligeable des études n'observe aucun effet, voire des effets bénéfiques sur l'adaptation au stress, y compris chez l'homme [9]. Vraisemblablement, le type de stress, son intensité et sa durée jouent un rôle dans les effets observés, de même que le sexe et les caractéristiques génétiques de l'individu qui est exposé au stress.

L'hypothèse selon laquelle l'environnement préconceptionnel et périnatal crée une empreinte sur l'individu est une problématique clé en psychobiologie du développement. Elle met en jeu des questions éthiques importantes [29] $(\rightarrow)$.

$\rightarrow$ Voir la Synthèse Comment les résultats des recherches sur la transmission intergénérationnelle du stress vontde $\varepsilon$. Rial-Sebbag et al., page 100 de ce numéro ils permettre d'améliorer la santé humaine? Une publication récente parue dans Science [30] montre que la stimulation cognitive et sociale précoce (entre 0 et 5 ans) chez des enfants exposés à un environnement socioéconomique défavorisé améliore significativement l'état de santé (marqueurs métaboliques, tension artérielle, poids) 30 ans plus tard. Ces résultats offrent une perspective intéressante en terme de politique publique. Comme le souligne le prix Nobel d'économie James Heckman, le retour sur investissement est maximal pour des prises en charges très précoces, entre 0 et 3 ans, puis, plus on s'éloigne de cette période critique, plus la balance coût-bénéfice se déséquilibre [31]. $\diamond$

\section{SUMMARY}

Early life stressful experiences and neuropsychiatric vulnerability: evidences from human and animal models

The human newborn is highly dependent on parental care for its survival but also for the healthy development of its brain. A large body of literature demonstrates the impact of early life adversity, even during the prenatal period, on the adult's health. The susceptibility to neuropsychiatric diseases is often potentiated by early stress. If there is an agreement that a critical developmental period exists, the mechanisms underlying the long term effects of early life adversity are still poorly understood. Recent studies in animals highlight the involvement of epigenetic processes in the transmission of such vulnerabilities, notably via modifications in germ cells, which can be transmitted in the next generations. $\diamond$

\section{LIENS D’INTÉRÊT}

Les auteurs déclarent n'avoir aucun lien d'intérêt concernant les données publiées dans cet article.

\section{RÉFÉRENCES}

1. McEwen BS. Understanding the potency of stressful early life experiences on brain and body function. Metabolism 2008; 57 (suppl 2) : S11-5.

2. Hackman DA, Farah MJ, Meaney MJ. Socioeconomic status and the brain: mechanistic insights from human and animal research. Nat Rev Neurosci $2010 ; 11: 651-9$.

3. McGowan PO, Sasaki A, D'Alessio AC, et al. Epigenetic regulation of the glucocorticoid receptor in human brain associates with childhood abuse. Nat Neurosci $2009 ; 12: 342-8$.

4. Provençal N, Binder $\varepsilon B$. The effects of early life stress on the epigenome: From the womb to adulthood and even before. Exp Neurol $2015 ; 268$ : $10-20$.

5. Levine $\mathrm{S}$. Infantile experience and resistance to physiological stress. Science $1957 ; 126: 405$.

6. Francis D, Diorio J, Liu D, et al. Nongenomic transmission across generations of maternal behavior and stress responses in the rat. Science $1999 ; 286$ : 1155-8.

7. Daugé $V$. Impact neurobiologique de la séparation mère/nouveau-né chez le rongeur. Med Sci (Paris) 2003 ; $19: 607-11$.

8. Vazquez V, Penit-Soria J, Durand C, et al. Maternal deprivation increases vulnerability to morphine dependence and disturbs the enkephalinergic system in adulthood. J Neurosci $2005 ; 25:$ 4453-62.

9. Franklin TB, Saab BJ, Mansuy IM. Neural mechanisms of stress resilience and vulnerability. Neuron $2012 ; 75: 747-61$.

10. Barreau F, Ferrier L, Fioramonti J, et al. New insights in the etiology and pathophysiology of irritable bowel syndrome: contribution of neonatal stress models. Pediatr Res $2007 ; 62: 240-5$.

11. Talge NM, Neal C, Glover V, et al. Antenatal maternal stress and long-term effects on child neurodevelopment: how and why? J Child Psychol Psychiatry $2007 ; 48: 245-61$

12. Joffe JM. Genotype and prenatal and premating stress interact to affect adult behavior in rats. Science $1965 ; 150: 1844-5$.

13. Darnaudéry M, Maccari $S$. Epigenetic programming of the stress response in male and female rats by prenatal restraint stress. Brain Res Rev 2008 ; 57 : 571-85.

14. Maccari S, Krugers HJ, Morley-Fletcher S, et al. The consequences of earlylife adversity: neurobiological, behavioural and epigenetic adaptations. J Neuroendocrinol $2014 ; 26: 707-23$.

15. Moisan MP, Le Moal M. Le stress dans tous ses états. Med Sci (Paris) 2012 ; $28: 612-7$.

16. Lemaire V, Koehl M, Le Moal M, et al. Prenatal stress produces learning deficits associated with an inhibition of neurogenesis in the hippocampus. Proc Natl Acad Sci USA. 2000 ; 97 : 11032-7.

17. Marrocco J, Mairesse J, Ngomba RT, et al. Anxiety-like behavior of prenatally stressed rats is associated with a selective reduction of glutamate release in the ventral hippocampus. J Neurosci $2012 ; 32: 17143-54$.

18. Weinstock M. Sex-dependent changes induced by prenatal stress in cortical and hippocampal morphology and behaviour in rats: an update. Stress Amst Neth $2011 ; 14: 604-13$. 


\section{RÉFÉRENCES}

19. Marrocco J, Reynaert $M-L$, Gatta $\varepsilon$, et al. The effects of antidepressant treatment in prenatally stressed rats support the glutamatergic hypothesis of stress-related disorders. J Neurosci 2014 ; $34: 2015-24$.

20. Morley-Fletcher S, Darnaudéry M, Mocaer $\varepsilon$, et al. Chronic treatment with imipramine reverses immobility behaviour, hippocampal corticosteroid receptors and cortical 5-HT(1A) receptor mRNA in prenatally stressed rats. Neuropharmacology $2004 ; 47: 841-7$.

21. Mairesse J, Lesage J, Breton C, et al. Maternal stress alters endocrine function of the fetoplacental unit in rats. Am J Physiol Endocrinol Metab 2007 ; 292 : ع1526-33.

22. Barbazanges A, Piazza PV, Le Moal M, et al. Maternal glucocorticoid secretion mediates long-term effects of prenatal stress. J Neurosci $1996 ; 16: 3943-9$.

23. Maccari S, Piazza PV, Kabbaj M, et al. Adoption reverses the long-term impairment in glucocorticoid feedback induced by prenatal stress. J Neurosci $1995 ; 15: 110-6$.

24. Weaver ICG, Cervoni N, Champagne FA, et al. Epigenetic programming by maternal behavior. Nat Neurosci $2004 ; 7: 847-54$.

25. Tesone-Coelho C, Morel LJ, Bhatt J, et al. Vulnerability to opiate intake in maternally deprived rats: implication of MeCP2 and of histone acetylation. Addict Biol $2015 ; 20: 120-31$.
26. Labonté B, Suderman M, Maussion G, et al. Genome-wide epigenetic regulation by early-life trauma. Arch Gen Psychiatry $2012 ; 69: 722-31$

27. Bock J, Wainstock T, Braun K, et al. Stress in utero: prenatal programming of brain plasticity and cognition. Biol Psychiatry $2015 ; 78: 315-26$.

28. Bale TL. Epigenetic and transgenerational reprogramming of brain development. Nat Rev Neurosci 2015 ; $16: 332-44$.

29. Richardson SS, Daniels CR, Gillman MW, et al. Society: don't blame the mothers. Nature $2014 ; 512$ : 131-2

30. Campbell F, Conti G, Heckman JJ, et al. Early childhood investments substantially boost adult health. Science $2014 ; 343: 1478-85$.

31. Heckman JJ. Schools, skills, and synapses. Econ Inq $2008 ; 46: 289$

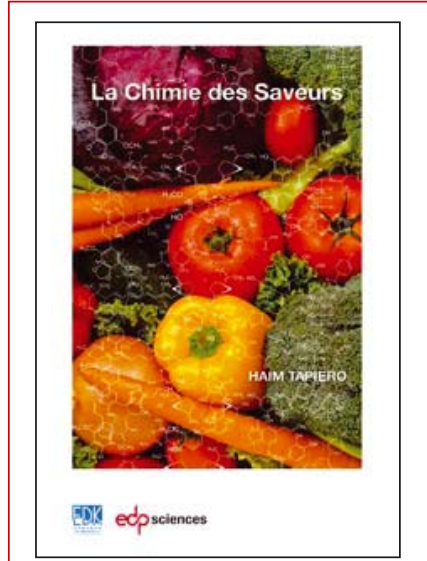

ISBN : 978-2-7598-1137-3 180 pages a cuisine est une science. II existe une relation étroite entre élaborer une recette et entreprendre une recherche scientifique. Quelle que soit l'origine d'une recette, d'un livre ou inventée, il faudra faire le choix des ingrédients, les mélanger et les cuire de manière appropriée afin de ne pas altérer les substances actives qui composent les ingrédients.

Une fois la cuisson terminée, il faudra analyser le goût et si nécessaire prévoir son amélioration. Améliorer une recette nécessite de connaître le ou les processus qui interviennent dans le développement des arômes, des saveurs et de la texture. Cette approche est similaire à celle développée par le scientifique.

La relation entre l'élaboration des recettes, les substances nutritives qui composent les ingrédients et la santé de l'homme est issue de plusieurs disciplines de la recherche fondamentale et clinique. Au cours des dernières années, de nombreux travaux scientifiques ont été publiés sur le rôle de la nutrition et la réduction des risques dans les pathologies comme les maladies cardio-vasculaires ou les cancers.

Le but principal de cet ouvrage a été d'identifier la structure chimique des composants actifs des ingrédients utilisés en cuisine (légumes, herbes aromatiques, épices) et qui entrent dans la préparation des recettes pour « végétariens » et « omnivores».

À retourner à EDK, 109, avenue Aristide Briand, 92541 Montrouge Cedex - Tél. : 0141177405 - Fax : 0149850345 - E-mail : edk@edk.fr NOM : Prénom :

Adresse :

Code postal :

Ville :

Pays :

Fonction :

Je souhaite recevoir l'ouvrage La chimie des Saveurs : $20 €+3 €$ de port $=\mathbf{2 3} €$ TTC

en ................ exemplaire, soit un total de

$\ldots$

$\square$ Par chèque, à l'ordre de $\mathbf{E} \mathbf{D} \mathbf{K}$

$\square$ Par carte bancaire : $\quad \square$ Visa $\square$ Eurocard/Mastercard

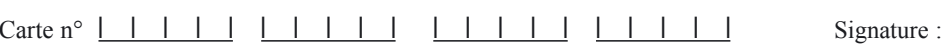

Date d'expiration :

$1 \quad 1 \quad 1 \quad 1 \quad$

$\mathrm{N}^{\circ}$ de contrôle au dos de la carte :

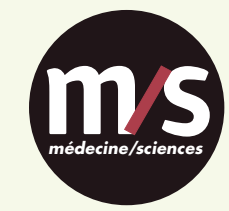

Tarifs d'abonnement $\mathrm{m} / \mathrm{s}-2016$

Abonnez-vous

à médecine/sciences
$>$ Grâce à $m / s$, vivez en direct les progrès des sciences biologiques et médicales

Bulletin d'abonnement page 130 dans ce numéro de $\mathrm{m} / \mathrm{s}$

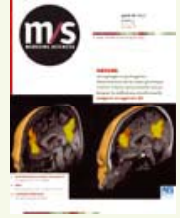

